\title{
Cellular Reprogramming: Recent Advances in Modeling Neurological Diseases
}

\author{
Guo-Li Ming, ${ }^{1,2,3}$ Oliver Brüstle, ${ }^{4}$ Alysson Muotri, ${ }^{5}$ Lorenz Studer, ${ }^{6}$ Marius Wernig, ${ }^{7}$ and Kimberly M. Christian ${ }^{1,2}$ \\ ${ }^{1}$ Institute for Cell Engineering, ${ }^{2}$ Department of Neurology, and ${ }^{3}$ The Solomon Snyder Department of Neuroscience, Johns Hopkins University School of \\ Medicine, Baltimore, Maryland 21205, ${ }^{4}$ Institute of Reconstructive Neurobiology, Life \& Brain Center, University of Bonn and Hertie Foundation, 53105 \\ Bonn, Germany, ${ }^{5}$ Departments of Pediatrics and Cellular \& Molecular Medicine, Stem Cell Program, University of California at San Diego, San Diego,

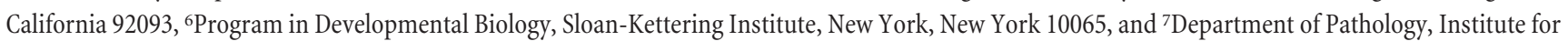 \\ Stem Cell Biology and Regenerative Medicine, Stanford University School of Medicine, Stanford, California 94305
}

The remarkable advances in cellular reprogramming have made it possible to generate a renewable source of human neurons from fibroblasts obtained from skin samples of neonates and adults. As a result, we can now investigate the etiology of neurological diseases at the cellular level using neuronal populations derived from patients, which harbor the same genetic mutations thought to be relevant to the risk for pathology. Therapeutic implications include the ability to establish new humanized disease models for understanding mechanisms, conduct high-throughput screening for novel biogenic compounds to reverse or prevent the disease phenotype, identify and engineer genetic rescue of causal mutations, and develop patient-specific cellular replacement strategies. Although this field offers enormous potential for understanding and treating neurological disease, there are still many issues that must be addressed before we can fully exploit this technology. Here we summarize several recent studies presented at a symposium at the 2011 annual meeting of the Society for Neuroscience, which highlight innovative approaches to cellular reprogramming and how this revolutionary technique is being refined to model neurodevelopmental and neurodegenerative diseases, such as autism spectrum disorders, schizophrenia, familial dysautonomia, and Alzheimer's disease.

\section{Introduction}

Recent advances in stem cell biology have led to technologies to reprogram somatic cells from the adult human to a state of pluripotency (Takahashi and Yamanaka, 2006; Takahashi et al., 2007; Yu et al., 2007; Park et al., 2008), or directly to neurons (Ambasudhan et al., 2011; Caiazzo et al., 2011; Pang et al., 2011; Pfisterer et al., 2011; Qiang et al., 2011; Yoo et al., 2011). The first reported lines of reprogrammed cells, termed induced pluripotent stem cells (iPSCs), were originally generated by introducing four transcription factors, including Oct3/4, Sox2, c-Myc, and $\mathrm{Klf} 4$, into mouse embryonic or adult fibroblasts using retroviruses (Takahashi and Yamanaka, 2006). When cultured under the same conditions used for embryonic stem cells, some of these

Received Aug. 15, 2011; revised Aug. 29, 2011; accepted Sept. 1, 2011.

This work was supported by the National Institutes of Health (NIH; NS048271, HD069184), National Alliance for Research on Schizophrenia and Depression, Maryland Stem Cell Research Fund (MSCRF), and Johns Hopkins Brain Science Institute to G-L.M.; by MSCRF to K.M.C.; by Deutsche Forschungsgemeinschaft Grant SFB TR3/D2, Bundesministerium für Bildung und Forschung Grants 01GN0813, 01GS0860, 01GN1008C, 01GN1009B, 0315799, 0315608A, and 0316020, European Union Grants 222943 and 266753, BI0.NRW Grant w0911bt027a, and the Hertie Foundation to 0.B.; by NIH (DPOD006395) and California Institute for Regenerative Medicine (CIRM) (TR2-01814) to A.M.; by New York State grants (N08S-005-01 NYSTEM, N09-G277-01 NYSTEM) and NIH (NS066390, NS072381, NS052671) to L.S.; and by NIH (MH092931 and NS073015), Ellison Medical Foundation Grant (AG-MS-0709-10), and CIRM (RT2-02061) to M.W. M.W. is an NYSCF-Robertson Investigator.

Correspondence should be addressed to either of the following: Dr. Guo-Li Ming, Institute for Cell Engineering, Department of Neurology, Johns Hopkins University School of Medicine, 733 North Broadway, BRB 779, Baltimore, MD 21205, E-mail: gming1@jhmi.edu; or Dr. Kimberly M. Christian, Institute for Cell Engineering, Department of Neurology, Johns Hopkins University School of Medicine, 733 North Broadway, BRB 775, Baltimore, MD 21205, E-mail: kchris12@jhmi.edu.

DOI:10.1523/JNEUROSCI.4218-11.2011

Copyright $\odot 2011$ the authors $\quad 0270-6474 / 11 / 3116070-06 \$ 15.00 / 0$ somatic cells were reprogrammed into cells exhibiting a pluripotent phenotype, expressing pluripotent markers and capable of differentiating into cells from the three germ layers. Subsequently, several groups demonstrated that this strategy can also be used to reprogram human fibroblasts into pluripotent iPSCs (Takahashi et al., 2007; Yu et al., 2007; Park et al., 2008). The field of cell reprogramming is moving forward at an unprecedented pace, with the development of many different reprogramming approaches. For example, ongoing research has begun to explore the use of different sets of reprogramming factors, application of chemical/small molecules to substitute for some genetic reprogramming factors, and foreign DNA integration-free methods (Juopperi et al., 2011). Furthermore, specific sets of reprogramming factors have been identified to reprogram somatic cells into a more restricted stem cell state, or even directly to a cell type originating from a different germ layer, such as from fibroblasts to neurons (Vierbuchen et al., 2010). Disease-specific iPSCs have also been derived from patients, and proof-of-principle studies have demonstrated the potential of using this special type of stem cell for therapeutic development (Juopperi et al., 2011).

These groundbreaking discoveries have redefined how we think about the modeling, treatment, and prevention of human diseases. Stem cell-based therapeutic strategies can be broadly categorized as follows: (1) a platform for the discovery of novel bioactive compounds through molecular dissection of the pathogenic process; or (2) a source for cellular replacement and tissue engineering through in vivo transplantation. On both of these fronts, much progress is being made, and the results will greatly 
enhance our understanding of the causal basis of many diseases. Advantages of using reprogrammed cells include the ability to investigate genetic contributions to cellular pathology with patient-specific cell sources, the generation of targeted cell populations, and the opportunity to model developmental pathology in a tractable and reproducible system. Despite the enormous promise, there are many challenges to be overcome before we can fully exploit the potential of this technology to engineer novel therapeutic strategies. The symposium will present the latest progress in the evolution of cellular reprogramming applications to understand disorders of the nervous system.

\section{Modeling neurodevelopmental disorders with a delayed onset} Dysregulation of neurodevelopmental processes can have profound and pervasive effects on the cognitive and physical abilities of affected individuals. Identifying the underlying etiology of these disorders can be extremely challenging due to the potential for a cascade of secondary effects that become difficult to dissociate from the primary pathology (Uhlhaas and Singer, 2010). The functional integrity of neural networks depends on the formation of effective and selective synaptic connections, and many of these networks are interdependent. Thus, the disruption of one neural system can lead to aberrant development in another. Likewise, mild perturbations of neuronal response properties can propagate during activity-driven development, resulting in a pervasive dysregulation. Exacerbating this complexity, many neurodevelopmental diseases are not linked to mutations at a single genetic locus. Instead, most disorders show a heritable degree of risk associated with multiple genetic loci, and this risk can be further modulated by epigenetic regulation and environmental factors (Keller and Persico, 2003; Harrison and Weinberger, 2005; Herbert, 2010). Derivation of iPSCs from patient fibroblasts provides a new platform to systematically investigate the genetic influence on disease pathogenesis.

Autism spectrum disorders (ASDs) comprise a wide range of neurodevelopmental diseases in which different combinations of genetic mutations may contribute to the symptoms (Geschwind, 2009; Weiss et al., 2009). Rett syndrome (RTT) is a rare example of an ASD in which a single X-linked gene, $M E C P 2$, has been identified as playing a causal role in the development of the disease (Amir et al., 1999). Because of the relatively straightforward genetic link, RTT is a useful model system to investigate cellular development in the context of mutations known to contribute to disease manifestation. Recently, Muotri and colleagues developed a culture system using iPSCs derived from RTT patients' fibroblasts (Marchetto et al., 2010). RTT patients' iPSCs are able to undergo X-inactivation and generate functional neurons. Neurons derived from RTT-iPSCs exhibited fewer synapses, reduced spine density, smaller soma size, altered calcium signaling, and electrophysiological defects when compared to controls in culture. Cumulative effects of altered spontaneous and activitydriven response properties suggest that network formation is compromised in RTT iPSC-derived cultures. Despite the variable expression of mutant $\mathrm{MeCP} 2$ due to X-inactivation, the defects were observed in all neurons differentiated from patient-derived iPSCs, indicating a non-cell-autonomous effect. One of the puzzling hallmarks of RTT is the symptomatological latency during early development. Typically, female RTT patients develop normally until 6-18 months of age, at which point they undergo developmental regression. By tracking the development of neuronal cultures derived from RTT patients, it was possible to identify a putative prodromal period in which a phenotypic disturbance is apparent at the cellular level during early neuronal development. These data thus provide evidence of an unexplored developmental window in RTT syndrome, before disease onset, when potential therapies could be successfully used and there can be a diagnostic readout of the efficacy of early intervention. To test the effects of drugs in rescuing synaptic defects, Muotri and colleagues investigated a nonspecific growth factor, IGF1, and observed a partial rescue of the RTT phenotype through an increase in synaptic number. Even more promising were the results from an antibiotic that impairs ribosomal proofreading, gentamicin, thus allowing for the expression of full-length MeCP2 levels in neurons with a nonsense mutation. This treatment restored the efficacy of glutamatergic synapses and may be a viable strategy for therapeutic intervention. By creating a model system based on a disease with a clear genetic association, they have developed an approach that can be applied to other ASD patientderived iPSCs to reveal common molecular and cellular pathways. Finally, some of the neuronal phenotypes described by the Muotri laboratory were independently confirmed (Kim et al., 2011), indicating that iPSCs derived from RTT patients can be a robust model to investigate this disease.

Schizophrenia (SZ) is a neuropsychiatric disorder with an even more prolonged latency of symptomatological onset, despite convergent evidence that suggests a neurodevelopmental origin of pathophysiology (Weinberger, 1987). Different from RTT, but similar to the vast majority of ASDs, schizophrenia is a complex genetic disorder with multiple identified risk factors. Among these, Disrupted-in-Schizophrenia 1 (DISC1) has emerged as a widely studied susceptibility gene, which plays a multifaceted role in neuronal development (St Clair et al., 1990; Millar et al., 2000; Ross et al., 2006; Duan et al., 2007; Kim et al., 2009; Mao et al., 2009). Ming and colleagues recently reported the derivation of iPSCs from siblings with a $4 \mathrm{bp}$ deletion in the DISC1 locus and a diagnosis of SZ (Chiang et al., 2011). Importantly, this study demonstrates the successful generation of adult patient-derived iPSCs using an episomal vector approach to avoid integration of foreign DNA into the host genome (Marchetto et al., 2009; Yu et al., 2009).

Reprogramming of somatic cells, such as dermal fibroblasts, was initially achieved through viral vectors, which carry the risk of insertional mutations, residual transgene expression, and tumorigenesis. Ideally, the induced state of pluripotency should resemble as closely as possible the endogenous state during human embryogenesis. One of the significant concerns in the field of iPSCs is whether and how the methods of induction are imprinted on the resultant stem cells (Gore et al., 2011; Hussein et al., 2011; Lister et al., 2011). Residual traces of the manipulation or epigenetic memory related to the donor cell may compromise the potential and quality of differentiation. Although some of these concerns may be independent of the transcription factor delivery strategy, episomal vectors do allow for integration-free reprogramming that minimizes the risk of many complications.

Patient-derived iPSCs will be enormously beneficial to the systematic investigation of complex genetic influences on disease risk and development. In conjunction with animal models used to identify the molecular pathways associated with risk genes, we can begin to evaluate the impact of genetic variability on cellular function and vulnerability to pathology. DISC1 is a prime example of a risk-associated gene, implicated in both SZ and ASD, that has been shown to regulate many aspects of neuronal development, including morphology, migration, and dendritic complexity in rodent models (Ming and Song, 2009; Christian et al., 2010). Although these data provide a compelling and plausible explanation of how this risk gene may contribute to developmen- 
tal pathology, there are still significant questions to be answered with respect to the human disease state. First, human DISC1 has multiple isoforms, and there is only an $\sim 60 \%$ homology with mouse Disc1 (Ma et al., 2002). Patient-derived iPSCs are an invaluable tool to create renewable populations of neurons that will harbor the same genetic mutations and may reveal novel functions of this gene in human neuronal development. Second, because SZ is a late-onset neurodevelopmental disease, there are outstanding questions regarding the role of environmental factors in triggering the emergence of symptoms. Investigating the development of neuronal populations with patient-specific mutations will allow us to identify compromised cellular functions that may render the neurons more vulnerable to environmental insult, which can be modeled in vitro. Third, an alternative, but not mutually exclusive, hypothesis of disease induction is that the presence of multiple genetic risk factors may take on causal properties that cannot be attributed to any single mutation. Because DISC1 is identified as a susceptibility gene in only a subset of patients with schizophrenia, it may be best understood as a contextual regulator of neuropathology. For example, DISC1 is central to a large interactome that has demonstrable effects on neuronal development (Camargo et al., 2007), and it is possible that DISC1 interacts synergistically with other genes and environmental factors to increase the effective risk for schizophrenia (Abazyan et al., 2010). In diseases with complex heritability and multiple identified risk factors, there is much work to be done to understand what constitutes the critical threshold for disease induction. Using DISC1 as a model to explore multivariate causes of disease-specific pathology, the generation of patient-derived iPSCs allows us to begin to document the role of genomic context in determining the impact of risk gene variation.

\section{Pharmaceutical and genetic rescue of neuronal phenotypes}

One striking recent example to demonstrate the potential of iPSCs in modeling human disease and screening of drug candidates comes from the generation of patient-derived neural crest lineages in familial dysautonomia (FD) (Lee and Studer, 2011). $\mathrm{FD}$ is a rare disorder in which a single point mutation in the I-k-B kinase complex-associated protein (IKBKAP) gene accounts for 99.5\% of all known cases. Symptoms of the disorder are severe gastrointestinal, respiratory, and cardiovascular problems and reflect pervasive autonomic neuron dysfunction. Using a lentiviral vector approach, Studer and colleagues have generated FD patient-derived CNS and PNS precursors, hematopoietic, endothelial, and endoderm cell types to investigate the extent of previously reported tissue-specific splicing (Slaugenhaupt et al., 2001). They did find evidence of tissue-specific differential IKBKAP splicing when compared to expression of WT transcript, but further work will be necessary to identify cell-type-specific splicing in the neural crest lineage. Interestingly, they also found a reduction in ASCL1 at the mRNA and protein level in the FDiPSC lines. Mash1, the mouse ortholog of ASCL1, has been shown to have a robust effect on neuronal development, and knock-out of this gene in mice prevents the generation of autonomic neurons (Guillemot et al., 1993). In FD-iPSC lines, ASCL1 ${ }^{+}$neurons differentiated from neural crest precursors revealed a significantly delayed time course of development, which appears consistent with the symptomatology in the human disease.

To investigate the potential for pharmaceutical rescue and putative therapeutic targets, Studer and colleagues screened a small set of candidate compounds identified in earlier studies using FD lymphoblast cell lines (Anderson et al., 2003a,b; Slaugenhaupt et al., 2004). Interestingly, the plant hormone kinetin was able to rescue several FD-iPSC phenotypes, including splicing and impaired production of autonomic neurons. Further analysis of treatment parameters should reveal novel information relevant for potential therapeutic application. Other options to rescue the phenotypes conferred by IKBKAP loss of function include genetic manipulation through $\mathrm{BAC}$ transgenesis or knock-in of the wild-type allele through homologous recombination. Because of the causative nature of the single point mutation, FD patient-derived iPSCs provide an ideal platform to develop advanced techniques for stem cell-based genetic rescue.

\section{Challenges for implementing reprogramming in drug development}

One of the most exciting new prospects provided by cell reprogramming is the possibility to conduct large-scale screens and develop therapeutic compounds directly on the neural cell types affected by the individual disease. The challenges associated with such an industrial application are significant. To screen hundreds of thousands of compounds, the systems used need to be scalable and robust enough to enable high-throughput analysis, including amenability to freezing and thawing as well as scalability in multitier formats suitable for automated processing. Considering that the readouts will be based on a comparison between cells derived from patients and healthy donors (or gene-corrected cells), the systems further need to be highly standardized. Ideally, one would like to define a standard population of expandable neural stem or progenitor cells, which can be derived from various pluripotent sources with little or no variability. At the same time, such a neural stem/progenitor cell population should still exhibit sufficient plasticity to be converted into different neuronal and glial cell types_- depending on the disease to be targeted by the approach. Finally, the population should be easily accessible to genetic modification, which is, e.g., crucial for equipping the cells with suitable reporter systems.

Brüstle and colleagues have recently succeeded in generating such a population from both human embryonic stem cells (hESCs) and iPSCs. These long-term self-renewing neuroepithelial-like stem cells (lt-NES cells) can be stably expanded across $>100$ passages while maintaining a stable neuronal and glial differentiation potential. They represent an early neuroepithelial stage as reflected by an ability to grow in neural rosette architectures. Neurons generated from lt-NES cells have been shown to interact synaptically with each other and, upon transplantation, with the host tissue. Most importantly, they retain some responsiveness to differentiation cues, and established combinations of morphogens can be used to induce midbrain dopamine and spinal cord motoneurons (Koch et al., 2009). Interestingly, the biological properties and gene expression patterns of lt-NES cells derived from different hESCs and iPSCs are remarkably similar-even if the pluripotent sources used for their generation show different propensities for neural differentiation. These observations suggest that lt-NES cells could serve as a kind of "standard" population for comparative studies on disease-specific and control cells (O. Brustle, unpublished work). Endowed with strong proliferation potential, lt-NES cells are easily amenable to genetic modification and subsequent selection-an advantage that circumvents the necessity of prior genetic modification and subsequent neural differentiation of pluripotent cells. Diseasespecific mutants can be directly introduced at the neural stem cell state, and the strong proliferation capacity readily enables the generation of bulk quantities of neurons and glia for biochemical studies and compound screening (O. Brustle, unpublished work). Although the generation of proliferative populations 
amenable to genetically introduced reporter systems is an essential first step, one of the significant challenges that lie ahead is the development of reliable and efficient methods to isolate specific populations of cells using approaches such as fluorescenceactivated cell sorting. Enrichment of targeted neuronal populations is critical for the investigation of disease-specific pathology, but there are also many outstanding questions as to how best to study these populations over time. It is likely that the critical insights from disease-specific models of neural pathology will be due to a convergence of information from long-term culture, transplantation, and an explicit investigation of cell-autonomous and non-cell-autonomous effects, all of which will depend on optimizing high-throughput strategies to generate relevant neuronal populations.

\section{Young neurons for modeling late-onset disorders?}

The majority of neurodegenerative disorders do not become apparent until late in life. In contrast, neural precursors, neurons, and glia derived from pluripotent cells typically represent early stages of nervous system development. To serve as a useful tool in modeling diseases of older age, human neuronal cells have to be deployed in a manner focused on pathogenic driver mechanisms rather than the end stage of neuronal degeneration. A common denominator in many neurodegenerative disorders is abnormal protein processing and aggregation. These changes may be based on genetic predisposition, such as expanded polyQ stretches in proteins affected in polyglutamine disorders. In these diseases, abnormal protein processing is a daily event, and it is only after years and decades of life that these changes finally induce degeneration of the neuronal target population affected by the disease. Thus, attempts to model established late-stage markers of degeneration, such as inclusion bodies, cytoskeletal changes, and neuronal death, using cell-based models might not be the most promising route. In this context, hESC and iPSC-derived neurons rather permit a developmental "replay" and molecular dissection of pathogenic driver mechanisms, providing a unique window of opportunity to study neurodegenerative diseases in a "presymptomatic" phase preceding neuronal degeneration.

\section{Direct programming as a shortcut to neuronal disease modeling}

An innovative approach with much potential to optimize the efficiency of generating neurons is the direct conversion of fibroblasts using defined transcription factors. In addition to increased efficiency, it may be possible to retain age-specific features of the source fibroblasts or accelerate the differentiation process, both of which could be helpful to expedite the investigation of aging-associated dysfunction (Chambers and Studer, 2011). Wernig and colleagues systematically investigated the potential of 19 genes known to play a role in neural development or epigenetic reprogramming and were able to identify a pool of five critical genes (Brn2, Myt1l, Ascl1, Zic1, and Olig2) with the potential to convert mouse fibroblasts to cells that behave like functional neurons (Vierbuchen et al., 2010). These induced neuronal (iN) cells not only expressed an array of neuronal markers and displayed a neuronal morphology but, importantly, possessed two principal functional properties of neurons: the ability to generate action potentials and the ability to form presynaptic and postsynaptic connections. Further investigation revealed that just three of those five factors are sufficient to induce fully functional iN cells: the basic helix loop helix transcription factor Ascll alone was sufficient to induce neuronal features, but the addition of Brn2 and Myt1l increased the efficiency of conversion and en- hanced the complexity of the neuronal properties. Neurons generated from direct reprogramming of either mouse embryonic or tail-tip fibroblasts were able to form functional synapses and generate trains of action potentials. The vast majority of these iN cells were glutamatergic, and the rest expressed markers consistent with an inhibitory or peripheral nervous system phenotype. In contrast to iPSC reprogramming, the conversion efficiencies were high (reaching 20\% in embryonic fibroblasts) and rapid. The first neuronal markers were expressed as early as $3 \mathrm{~d}$ after induction of the reprogramming factors and after 2 weeks, synaptically competent cells were identified. For translational applications, it is essential that this method also be effective in human cells. Somewhat surprisingly, the same factors that were sufficient to convert mouse fibroblasts were only sufficient to induce neuronal markers and gross neuronal morphology, but no convincing functional properties, using human fibroblasts. In a further systematic screen, another basic helix loop helix transcription factor, Neurod1, was identified that enabled the generation of functional iN cells from human embryonic and postnatal foreskin fibroblasts in conjunction with the three other factors (Pang et al., 2011). Similar to human ESC and iPSC differentiation systems, the maturation of neurons required extended periods of time in culture compared to mouse cells. This observation is consistent with the slower development of the human nervous system. Recently, these results were corroborated and extended by another group who found a synergistic effect of the expression of the microRNAs miR-9/miR-124 and the transcription factors Neurod2, Ascl1, and Myt1l in the presence of an HDAC inhibitor to facilitate the generation of iN cells from human neonatal and adult fibroblasts (Yoo et al., 2011). Demonstrating the applicability of direct reprogramming in a disease context, iN cells were also recently generated from fibroblasts obtained from familial and sporadic Alzheimer's patients using the five transcription factors initially identified in the mouse study (Qiang et al., 2011). Importantly, significantly altered production of amyloid- $\beta$ peptides was observed compared to control cells, illustrating the capacity of iN cells to retain disease traits. Rapid and efficient generation of patient-specific neuronal populations through direct reprogramming may ultimately yield many advantages in the screening of pharmaceutical compounds as well as providing a source of cellular material suitable for transplantation.

\section{Conclusions and future research}

The advent of cellular reprogramming has opened up vast new territory in the search for effective treatments of neurological disease. We have the opportunity to develop patient-specific treatments and begin to understand the genetic complexities underlying many neural disorders. Many of the challenges that were identified at the outset of this field of research are being directly addressed through the refinement of techniques and the development of innovative and novel approaches. Integration-free iPSCs and direct reprogramming may circumvent many of the complications arising from insertional mutagenesis and tumorigenic properties of pluripotent stem cells. Modeling of monogenic diseases, such as FD and RTT, may provide the most straightforward path to developing high-throughput screens of novel bioactive compounds for therapeutic intervention. In addition, FD and RTT patient-derived iPSCs can facilitate the development of advanced techniques for genetic rescue of disease phenotypes. In combination with whole-genome analysis of individual patients, SZ patient-derived iPSCs may provide an excellent model to explore how prominent risk genes functionally interact to result in a suprathreshold level of cellular vulnerability 
that leads to disease induction. Recapitulation of neuronal development in a rigorous and reproducible system will also allow for the targeted investigation of the impact of environmental factors on cellular function. High-throughput screening of potential therapeutic compounds will greatly benefit from the recent generation of a population of neuroepithelial-like stem cells, which provides a standardized and renewable cellular source to generate disease-relevant neuronal populations. Together, these recent advancements in cellular reprogramming have made it possible to approach human disease modeling using relevant human cell types. Studies of induced human neurons from patients, generated through either direct conversion or derived from induced pluripotent stem cells, will give us valuable insight into disease mechanisms and will facilitate cellular replacement and drug discovery.

\section{References}

Abazyan B, Nomura J, Kannan G, Ishizuka K, Tamashiro KL, Nucifora F, Pogorelov V, Ladenheim B, Yang C, Krasnova IN, Cadet JL, Pardo C, Mori S, Kamiya A, Vogel MW, Sawa A, Ross CA, Pletnikov MV (2010) Prenatal interaction of mutant DISC1 and immune activation produces adult psychopathology. Biol Psychiatry 68:1172-1181.

Ambasudhan R, Talantova M, Coleman R, Yuan X, Zhu S, Lipton SA, Ding S (2011) Direct reprogramming of adult human fibroblasts to functional neurons under defined conditions. Cell Stem Cell 9:113-118.

Amir RE, Van den Veyver IB, Wan M, Tran CQ, Francke U, Zoghbi HY (1999) Rett syndrome is caused by mutations in X-linked MECP2, encoding methyl-CpG-binding protein 2. Nat Genet 23:185-188.

Anderson SL, Qiu J, Rubin BY (2003a) EGCG corrects aberrant splicing of IKAP mRNA in cells from patients with familial dysautonomia. Biochem Biophys Res Commun 310:627-633.

Anderson SL, Qiu J, Rubin BY (2003b) Tocotrienols induce IKBKAP expression: a possible therapy for familial dysautonomia. Biochem Biophys Res Commun 306:303-309.

Caiazzo M, Dell'Anno MT, Dvoretskova E, Lazarevic D, Taverna S, Leo D, Sotnikova TD, Menegon A, Roncaglia P, Colciago G, Russo G, Carninci P, Pezzoli G, Gainetdinov RR, Gustincich S, Dityatev A, Broccoli V (2011) Direct generation of functional dopaminergic neurons from mouse and human fibroblasts. Nature 476:224-227.

Camargo LM, Collura V, Rain JC, Mizuguchi K, Hermjakob H, Kerrien S, Bonnert TP, Whiting PJ, Brandon NJ (2007) Disrupted in Schizophrenia 1 interactome: evidence for the close connectivity of risk genes and a potential synaptic basis for schizophrenia. Mol Psychiatry 12:74-86.

Chambers SM, Studer L (2011) Cell fate plug and play: direct reprogramming and induced pluripotency. Cell 145:827-830.

Chiang CH, Su Y, Wen Z, Yoritomo N, Ross CA, Margolis RL, Song H, Ming GL (2011) Integration-free induced pluripotent stem cells derived from schizophrenia patients with a DISCl mutation. Mol Psychiatry 16:358-360.

Christian K, Song H, Ming GL (2010) Adult neurogenesis as a cellular model to study schizophrenia. Cell Cycle 9:636-637.

Duan X, Chang JH, Ge S, Faulkner RL, Kim JY, Kitabatake Y, Liu XB, Yang CH, Jordan JD, Ma DK, Liu CY, Ganesan S, Cheng HJ, Ming GL, Lu B, Song H (2007) Disrupted-In-Schizophrenia 1 regulates integration of newly generated neurons in the adult brain. Cell 130:1146-1158.

Geschwind DH (2009) Advances in autism. Annu Rev Med 60:367-380.

Gore A, Li Z, Fung HL, Young JE, Agarwal S, Antosiewicz-Bourget J, Canto I, Giorgetti A, Israel MA, Kiskinis E, Lee JH, Loh YH, Manos PD, Montserrat N, Panopoulos AD, Ruiz S, Wilbert ML, Yu J, Kirkness EF, Izpisua Belmonte JC, et al. (2011) Somatic coding mutations in human induced pluripotent stem cells. Nature 471:63-67.

Guillemot F, Lo LC, Johnson JE, Auerbach A, Anderson DJ, Joyner AL (1993) Mammalian achaete-scute homolog 1 is required for the early development of olfactory and autonomic neurons. Cell 75:463-476.

Harrison PJ, Weinberger DR (2005) Schizophrenia genes, gene expression, and neuropathology: on the matter of their convergence. Mol Psychiatry 10:40-68; image 5 .

Herbert MR (2010) Contributions of the environment and environmentally vulnerable physiology to autism spectrum disorders. Curr Opin Neurol 23:103-110.
Hussein SM, Batada NN, Vuoristo S, Ching RW, Autio R, Närvä E, Ng S, Sourour M, Hämäläinen R, Olsson C, Lundin K, Mikkola M, Trokovic R, Peitz M, Brüstle O, Bazett-Jones DP, Alitalo K, Lahesmaa R, Nagy A, Otonkoski T (2011) Copy number variation and selection during reprogramming to pluripotency. Nature 471:58-62.

Juopperi TA, Song H, Ming GL (2011) Modeling neurological diseases using patient-derived induced pluripotent stem cells. Future Neurol 6:363-373.

Keller F, Persico AM (2003) The neurobiological context of autism. Mol Neurobiol 28:1-22.

Kim JY, Duan X, Liu CY, Jang MH, Guo JU, Pow-anpongkul N, Kang E, Song H, Ming GL (2009) DISC1 regulates new neuron development in the adult brain via modulation of AKT-mTOR signaling through KIAA1212. Neuron 63:761-773.

Kim KY, Hysolli E, Park IH (2011) Neuronal maturation defect in induced pluripotent stem cells from patients with Rett syndrome. Proc Natl Acad Sci U S A 108:14169-14174.

Koch P, Opitz T, Steinbeck JA, Ladewig J, Brüstle O (2009) A rosette-type, self-renewing human ES cell-derived neural stem cell with potential for in vitro instruction and synaptic integration. Proc Natl Acad Sci U S A 106:3225-3230

Lee G, Studer L (2011) Modelling familial dysautonomia in human induced pluripotent stem cells. Philos Trans R Soc Lond B Biol Sci 366:2286-2296.

Lister R, Pelizzola M, Kida YS, Hawkins RD, Nery JR, Hon G, AntosiewiczBourget J, O’Malley R, Castanon R, Klugman S, Downes M, Yu R, Stewart R, Ren B, Thomson JA, Evans RM, Ecker JR (2011) Hotspots of aberrant epigenomic reprogramming in human induced pluripotent stem cells. Nature 471:68-73.

Ma L, Liu Y, Ky B, Shughrue PJ, Austin CP, Morris JA (2002) Cloning and characterization of Disc1, the mouse ortholog of DISC1 (Disrupted-inSchizophrenia 1). Genomics 80:662-672.

Mao Y, Ge X, Frank CL, Madison JM, Koehler AN, Doud MK, Tassa C, Berry EM, Soda T, Singh KK, Biechele T, Petryshen TL, Moon RT, Haggarty SJ, Tsai LH (2009) Disrupted in schizophrenia 1 regulates neuronal progenitor proliferation via modulation of GSK3beta/beta-catenin signaling. Cell 136:1017-1031.

Marchetto MC, Yeo GW, Kainohana O, Marsala M, Gage FH, Muotri AR (2009) Transcriptional signature and memory retention of humaninduced pluripotent stem cells. PLoS One 4:e7076

Marchetto MC, Carromeu C, Acab A, Yu D, Yeo GW, Mu Y, Chen G, Gage FH, Muotri AR (2010) A model for neural development and treatment of Rett syndrome using human induced pluripotent stem cells. Cell 143:527-539.

Millar JK, Wilson-Annan JC, Anderson S, Christie S, Taylor MS, Semple CA, Devon RS, St Clair DM, Muir WJ, Blackwood DH, Porteous DJ (2000) Disruption of two novel genes by a translocation co-segregating with schizophrenia. Hum Mol Genet 9:1415-1423.

Ming GL, Song H (2009) DISC1 partners with GSK3beta in neurogenesis. Cell 136:990-992.

Pang ZP, Yang N, Vierbuchen T, Ostermeier A, Fuentes DR, Yang TQ, Citri A, Sebastiano V, Marro S, Südhof TC, Wernig M (2011) Induction of human neuronal cells by defined transcription factors. Nature 476:220-223.

Park IH, Zhao R, West JA, Yabuuchi A, Huo H, Ince TA, Lerou PH, Lensch MW, Daley GQ (2008) Reprogramming of human somatic cells to pluripotency with defined factors. Nature 451:141-146.

Pfisterer U, Kirkeby A, Torper O, Wood J, Nelander J, Dufour A, Björklund A, Lindvall O, Jakobsson J, Parmar M (2011) Direct conversion of human fibroblasts to dopaminergic neurons. Proc Natl Acad Sci USA 108:10343-10348.

Qiang L, Fujita R, Yamashita T, Angulo S, Rhinn H, Rhee D, Doege C, Chau L, Aubry L, Vanti WB, Moreno H, Abeliovich A (2011) Directed conversion of Alzheimer's disease patient skin fibroblasts into functional neurons. Cell 146:359-371.

Ross CA, Margolis RL, Reading SA, Pletnikov M, Coyle JT (2006) Neurobiology of schizophrenia. Neuron 52:139-153.

Slaugenhaupt SA, Blumenfeld A, Gill SP, Leyne M, Mull J, Cuajungco MP, Liebert CB, Chadwick B, Idelson M, Reznik L, Robbins C, Makalowska I, Brownstein M, Krappmann D, Scheidereit C, Maayan C, Axelrod FB, Gusella JF (2001) Tissue-specific expression of a splicing mutation in the IKBKAP gene causes familial dysautonomia. Am J Hum Genet 68:598-605.

Slaugenhaupt SA, Mull J, Leyne M, Cuajungco MP, Gill SP, Hims MM, Quin- 
tero F, Axelrod FB, Gusella JF (2004) Rescue of a human mRNA splicing defect by the plant cytokinin kinetin. Hum Mol Genet 13:429-436.

St Clair D, Blackwood D, Muir W, Carothers A, Walker M, Spowart G, Gosden C, Evans HJ (1990) Association within a family of a balanced autosomal translocation with major mental illness. Lancet 336:13-16.

Takahashi K, Yamanaka S (2006) Induction of pluripotent stem cells from mouse embryonic and adult fibroblast cultures by defined factors. Cell 126:663-676.

Takahashi K, Tanabe K, Ohnuki M, Narita M, Ichisaka T, Tomoda K, Yamanaka S (2007) Induction of pluripotent stem cells from adult human fibroblasts by defined factors. Cell 131:861-872.

Uhlhaas PJ, Singer W (2010) Abnormal neural oscillations and synchrony in schizophrenia. Nat Rev Neurosci 11:100-113.

Vierbuchen T, Ostermeier A, Pang ZP, Kokubu Y, Südhof TC, Wernig M (2010) Direct conversion of fibroblasts to functional neurons by defined factors. Nature 463:1035-1041.
Weinberger DR (1987) Implications of normal brain development for the pathogenesis of schizophrenia. Arch Gen Psychiatry 44:660-669.

Weiss LA, Arking DE, Daly MJ, Chakravarti A (2009) A genome-wide linkage and association scan reveals novel loci for autism. Nature 461:802-808.

Yoo AS, Sun AX, Li L, Shcheglovitov A, Portmann T, Li Y, Lee-Messer C, Dolmetsch RE, Tsien RW, Crabtree GR (2011) MicroRNAmediated conversion of human fibroblasts to neurons. Nature 476 : $228-231$.

Yu J, Vodyanik MA, Smuga-Otto K, Antosiewicz-Bourget J, Frane JL, Tian S, Nie J, Jonsdottir GA, Ruotti V, Stewart R, Slukvin II, Thomson JA (2007) Induced pluripotent stem cell lines derived from human somatic cells. Science 318:1917-1920.

Yu J, Hu K, Smuga-Otto K, Tian S, Stewart R, Slukvin II, Thomson JA (2009) Human induced pluripotent stem cells free of vector and transgene sequences. Science 324:797-801. 